\title{
One in five hospitalized children is malnourished
}

\author{
Cite as: CMAJ 2019 July 15;191:E798. doi: 10.1503/cmaj.109-5772
}

Posted on cmajnews.com on June 24, 2019.

M any Canadian children are malnourished and lose weight in hospital without doctors recognizing the problem, experts warned at the Canadian Paediatric Society's annual meeting. They're urging health teams to screen for malnutrition on admission like they do for infectious diseases.

A recent Canadian multicentre trial found that one in five children is malnourished on admission to hospital. Only half who were malnourished or at high risk of malnutrition saw a dietician. Study coauthor Dr. Valérie Marchand, a professor in the pediatrics department at the Université de Montréal, said the results are "really worrisome" and suggested that many children may not be receiving appropriate treatment.

Children who were well-nourished on admission and those who didn't see a dietician in hospital were more likely to lose weight during their stay, probably because "nobody really focused on their nutrition." Kids with underlying or severe medical conditions faced higher risks of malnutrition. Often this can become a vicious cycle, Marchand noted.

"We have to work even harder to feed those patients because malnutrition will increase morbidity, decrease wound healing, increase the risk of infection and complications, and slow down recovery."

That means more deaths, longer hospital stays, increased costs, and lower quality of life for young patients. Screening for malnutrition on admission to hospital could reduce these harms, but few hospitals do it, Marchand said.

If no one is paying attention, it's easy for a child to become malnourished in hospital. Many young patients will eat less because of

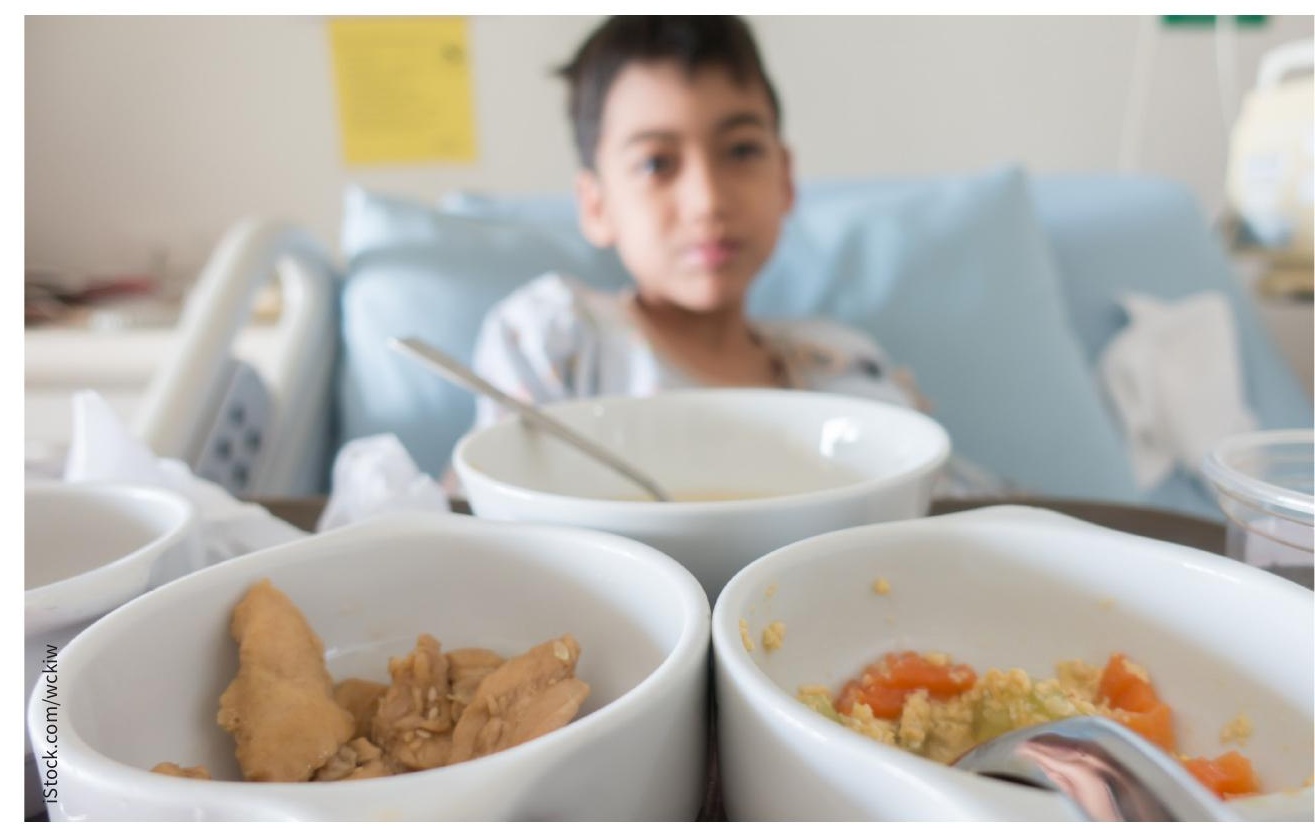

Few hospitals screen children for malnutrition.

stress or may not be able to communicate their needs to nursing staff without their parents' help. Sometimes parents finish their kids' meals without staff knowing. Also, doctors may order tests that require fasting, which can worsen a child's condition if those tests are delayed.

Children who are overweight are also often overlooked, Marchand added. She recently saw a patient with inflammatory bowel disease who lost 30 pounds "and people didn't take it seriously because he was still a little chubby." As it turned out, he was malnourished.

Nutritional screening should be done on all patients and should be easy to implement, Marchand said. However, she acknowledged that many doctors lack the time and resources; even her own institution doesn't do it. "Those are the things that we need to work on."
Heather Keller, Schlegel Research Chair of Nutrition and Aging at the University of Waterloo, said pediatric units can learn from projects to identify malnutrition in adults. Her study of the More-2-Eat project at five hospitals across Canada demonstrated that nutritional screening and diagnosis is feasible and improves care. In the first phase of the project, the sites achieved a $70 \%-75 \%$ screening rate. By the second phase, all severely malnourished patients received a comprehensive nutritional assessment.

Educating staff about malnutrition, embedding screening into electronic medical records, and providing feedback on performance were key to the project's success, said Keller. "Given the capability and motivation, they will change their behaviour."

Lauren Vogel, CMAJ 